\title{
Atomic Sequential Effect Algebras
}

\author{
Josef Tkadlec
}

Received: 30 November 2006 / Accepted: 12 July 2007 / Published online: 7 August 2007

\begin{abstract}
Various conditions ensuring that a sequential effect algebra or the set of sharp elements of a sequential effect algebra is a Boolean algebra are presented.
\end{abstract}

Keywords Effect algebra · Boolean algebra - Sequential product · Atomic $\cdot$ Sharp

The basic algebraic object for studying quantum structures is the effect algebra - see, e.g., Foulis and Bennet [2]. Gudder (see, e.g., [5]) introduced the notion of a sequential product on an effect algebra as an abstract formalization of a sequential measurement. Sequential effect algebras have the property that sharp elements remain sharp whenever we use an embedding to a greater structure, hence the notion of sharpness is not contextual within sequential effect algebras. In this paper we present several results stating that a sequential effect algebra (or the set of sharp elements of a sequential effect algebra) is a Boolean algebra. Some of them are generalizations of analogous results of Gudder and Greechie [5].

\section{Basic Notions}

Definition 1.1 An effect algebra is an algebraic structure $(E, \oplus, 0,1)$ such that $E$ is a set, 0 and 1 are different elements of $E$ and $\oplus$ is a partial binary operation on $E$ such that for every $a, b, c \in E$ the following conditions hold (the equalities mean also "if one side exists then the other side exists"):

(1) $a \oplus b=b \oplus a$ (commutativity),

(2) $(a \oplus b) \oplus c=a \oplus(b \oplus c)$ (associativity),

(3) for every $a \in E$ there is a unique $a^{\prime} \in E$ such that $a \oplus a^{\prime}=1$ (orthosupplement),

J. Tkadlec

Department of Mathematics, Faculty of Electrical Engineering, Czech Technical University,

16627 Prague, Czech Republic

e-mail: tkadlec@fel.cvut.cz 
(4) $a=0$ whenever $a \oplus 1$ is defined (zero-unit law).

For simplicity, we use the notation $E$ for an effect algebra. A partial ordering on an effect algebra $E$ is defined by $a \leq b$ iff there is a $c \in E$ such that $b=a \oplus c$; such an element $c$ is unique (if it exists) and is denoted by $b \ominus a$. Also, 0 (1, resp.) is the least (the greatest, resp.) element of $E$ with respect to this partial ordering. An orthogonality relation on $E$ is defined by $a \perp b$ iff $a \oplus b$ exists (i.e., iff $a \leq b^{\prime}$ ). It can be shown that $a \oplus 0=a$ for every $a \in E$ and that the cancellation law is valid: for every $a, b, c \in E$ with $a \oplus b \leq a \oplus c$ we have $b \leq c$.

For $a \leq b$ we denote $[a, b]=\{c \in E: a \leq c \leq b\}$. A chain in $E$ is a nonempty linearly (totally) ordered subset of $E$.

Definition 1.2 Let $E$ be an effect algebra. An element $a \in E$ is called

- sharp, if $a \wedge a^{\prime}=0$;

- principal, if $b \oplus c \leq a$ for every $b, c \in E$ with $b \perp c$ and $b, c \leq a$;

- central, if $a$ and $a^{\prime}$ are principal and for every $b \in E$ there are $b_{1}, b_{2} \in E$ such that $b_{1} \leq a, b_{2} \leq a^{\prime}$ and $b=b_{1} \oplus b_{2}$. The set of central elements of $E$ is called the center of E.

By definition, every central element is principal, and it is well-known and easy to see that every principal element is sharp. The reverse implications need not be true.

Definition 1.3 An orthoalgebra is an effect algebra in which every its element is sharp.

An orthomodular poset is an effect algebra in which every its element is principal.

Since every principal element in an effect algebra is sharp, every orthomodular poset is an orthoalgebra.

\section{Atomic Effect Algebras}

Definition 2.1 Let $E$ be an effect algebra.

- An atom in $E$ is a minimal element of $E \backslash\{0\}$.

- The effect algebra $E$ is atomic if every nonzero element of $E$ dominates an atom.

- The effect algebra $E$ is atomistic if every nonzero element of $E$ is a supremum of a set of atoms (hence dominated by this element).

- The effect algebra $E$ is determined by atoms if for different elements $a, b \in E$ the sets of atoms in $[0, a]$ and $[0, b]$ are different.

Let us remark that an effect algebra is atomistic iff the atoms are order determining in the sense that, if every atom in $[0, a]$ belongs to $[0, b]$, then $a \leq b$.

Lemma 2.2 Every atomistic effect algebra is determined by atoms. Every effect algebra determined by atoms is atomic.

Proof Let $E$ be an atomistic effect algebra. Since every nonzero element of $E$ is the supremum of atoms it dominates, for different elements we obtain different sets of dominated atoms. 
Let $E$ be an effect algebra determined by atoms. Then for every nonzero element $a \in E$ the set of dominated atoms is nonempty.

Greechie 3 presented examples of atomic orthomodular posets not determined by atoms. Let us present an example of a nonatomistic orthomodular poset determined by atoms.

Example 2.3 Let $X_{1}=\left\{x_{1}\right\}, X_{2}=\left\{x_{2}\right\}, X_{3}$ and $X_{4}$ be mutually disjoint sets, and let $X_{3}, X_{4}$ be infinite. Let us put $X=\bigcup_{i=1}^{4} X_{i}$,

$$
\begin{aligned}
E^{\prime} & =\left\{\emptyset, X_{1} \cup X_{2}, X_{2} \cup X_{3}, X_{3} \cup X_{4}, X_{4} \cup X_{1}, X\right\}, \\
E & =\left\{(A \backslash F) \cup(F \backslash A): A \in E^{\prime} \text { and } F \subset X_{3} \cup X_{4} \text { is finite }\right\} .
\end{aligned}
$$

Then $(E, \oplus, \emptyset, X)$ with $A \oplus B=A \cup B$ for disjoint $A, B \in E$ is an orthomodular poset. The orthosupplement is the set theoretic complement in $X$, the partial ordering is the inclusion. The atoms in $E$ are $\left\{x_{1}, x_{2}\right\}$ and one-element subsets of $X_{3} \cup X_{4}$.

$E$ is not atomistic because the set of atoms dominated by the element $X_{1} \cup X_{4}$ is the set of one-element subsets of $X_{4}$ that has $X_{3} \cup X_{4}$ as an upper bound and $X_{3} \cup X_{4} ¥ X_{1} \cup X_{4}$.

Let us prove that $E$ is determined by atoms. Let $A, B \in E$ such that the sets of atoms dominated by $A$ and $B$ coincide. Since $\{x\}$ is an atom for every $x \in X_{3} \cup X_{4}$, we obtain $A \cap\left(X_{3} \cup X_{4}\right)=B \cap\left(X_{3} \cup X_{4}\right)$. Let us suppose that $A \neq B$ and seek a contradiction. Then, e.g., $A \not \subset B$ and there is an $x \in X_{1} \cup X_{2}$ such that $x \in A \backslash B$. Let, e.g., $x=x_{1}$. Since $\left\{x_{1}, x_{2}\right\}$ is an atom not dominated by $B$, it is not dominated by $A$ and therefore $x_{2} \notin A$. Hence $B \cap X_{4}=A \cap X_{4}$ is cofinite and $B \cap X_{3}=A \cap X_{3}$ is finite. Therefore $x_{1} \in B$-a contradiction.

It is known that every atomic orthomodular lattice is atomistic - see, e.g., Pták and Pulmannová [6. Let us present an analogous result for effect algebras (lattice orthoalgebras are orthomodular lattices).

Proposition 2.4 Every lattice effect algebra determined by atoms is atomistic.

Proof Let $E$ be a lattice effect algebra determined by atoms, $a \in E \backslash\{0\}$ and $A$ be the set of atoms in $[0, a]$. For every $b \in E$ that dominates all elements of $A$ we obtain that $A$ is the set of atoms in $[0, a \wedge b]$ and therefore, since $E$ is determined by atoms, $a=a \wedge b \leq b$. Hence $a=\bigvee A$.

Let us remark that for example the 3 -chain $C_{3}=\{0, a, 1\}$ with $a \oplus a=1$ and $x \oplus 0=x$ for every $x \in C_{3}$ is an atomic lattice effect algebra that is not determined by atoms - different elements $a, 1$ dominate the same set $\{a\}$ of atoms.

Proposition 2.5 Every effect algebra in which every its nonzero element dominates a nonzero sharp element is an orthoalgebra.

Proof Let us suppose that the effect algebra $E$ is not an orthoalgebra and seek a contradiction. There is an unsharp element $a \in E$. Hence there is a nonzero element $b \in E$ such that $b \leq a, a^{\prime}$. According to the assumption, there is a nonzero sharp element $c \in E$ such that $c \leq b$. Then $c \leq a, a^{\prime}$ and therefore $a \leq c^{\prime}$. Hence $c \leq a \leq c^{\prime}$ and therefore $c \wedge c^{\prime}=c \neq 0-\mathrm{a}$ contradiction. 
Corollary 2.6 Every atomic effect algebra in which every its atom is sharp is an orthoalgebra.

\section{Sequential Effect Algebras}

Definition 3.1 A sequential product on an effect algebra $E$ is a binary operation $\circ$ on $E$ such that for every $a, b, c \in E$ the following conditions hold:

(1) $a \circ(b \oplus c)=(a \circ b) \oplus(a \circ c)$ if $b \oplus c$ exists;

(2) $1 \circ a=a$;

(3) if $a \circ b=0$ then $a \mid b$ (where $a \mid b$ denotes $a \circ b=b \circ a)$;

(4) if $a \mid b$ then $a \mid b^{\prime}$ and $a \circ(b \circ c)=(a \circ b) \circ c$;

(5) if $c \mid a, b$ then $c \mid a \circ b$ and $c \mid a \oplus b$ (if $a \oplus b$ exists).

An effect algebra with a sequential product is called a sequential effect algebra.

For examples of sequential effect algebras see Gudder and Greechie [5]-e.g., every Boolean algebra with $a \circ b=a \wedge b$ forms a sequential effect algebra, the set of positive self-adjoint operators on a Hilbert space bounded by the identity with $A \circ B=A^{1 / 2} B A^{1 / 2}$ forms a sequential algebra and there is an atomic sequential effect algebra that is not a Boolean algebra (Sect. 7).

Let us present some results concerning sequential effect algebras.

Proposition 3.2 Let $E$ be a sequential effect algebra. Then for every $a, b \in E$ the following properties hold:

(1) $a \circ 0=0 \circ a=0$;

(2) $a \circ 1=1 \circ a=a$;

(3) $a \circ b \leq a$;

(4) if $a$ is sharp then $a \leq b$ iff $a \circ b=b \circ a=a$.

Proof See Gudder and Greechie [5, Lemma 3.1 and Theorem 3.4.

Proposition 3.3 Let $E$ be a sequential effect algebra, $a \in E$ be an atom. Then $a \mid b$ for every $b \in E$.

Proof Let $b \in E$. Since $a \circ b \leq a$ and $a$ is an atom, we obtain that $a \circ b \in\{0, a\}$. If $a \circ b=0$ then $a \mid b$ from the definition of a sequential product. Let us suppose that $a \circ b=a$. We obtain that $a \circ b=a=a \circ 1=a \circ\left(b \oplus b^{\prime}\right)=(a \circ b) \oplus\left(a \circ b^{\prime}\right)$ and therefore $a \circ b^{\prime}=0$. Hence $a \mid b^{\prime}$ and therefore $a \mid b$.

Lemma 3.4 Let $E$ be a sequential effect algebra, $a, b, c \in E$ such that $a$ is sharp, $a \mid c$ and $a \leq b \circ c$. Then $a \leq b, c$. If, moreover, $a \mid(c \circ b)$ then $a \leq c \circ b$.

Proof According to Proposition 3.2, $a \leq b \circ c \leq b$ and, since $a$ is sharp, $a \mid b$. Since $a \leq b, b \circ c, a$ is sharp and $a \mid b, c$, we obtain, according to Proposition 3.2 and the definition of a sequential product, that $a=a \circ(b \circ c)=(a \circ b) \circ c=a \circ c=c \circ a \leq c$. Hence, if moreover $a \mid(c \circ b)$, we obtain that $a=a \circ b=(a \circ c) \circ b=a \circ(c \circ b)=(c \circ b) \circ a \leq c \circ b$. 
Proposition 3.5 Let $E$ be a sequential effect algebra, $a \in E$ be a sharp atom. Then $a \leq b \circ c$ iff $a \leq c \circ b$ for every $b, c \in E$.

Proof Let $b, c \in E$ such that $a \leq b \circ c$. According to Proposition 3.3 $a \mid c$ and $a \mid(c \circ b)$. According to Lemma 3.4, $a \leq c \circ b$. The reverse implication can be proved analogously.

Let us summarize some properties of sequential effect algebras that we will use in the sequel.

Proposition 3.6 Let $E$ be a sequential effect algebra.

(1) The set of sharp elements of $E$ is a sub-effect algebra and forms an orthomodular poset.

(2) Let $a, b \in E$ be sharp. If $a \wedge b$ (resp., $a \vee b)$ exists in $E$ then $a \wedge b$ is sharp (resp., $a \vee b$ is sharp).

(3) If $E$ is chain finite then every atom of $E$ is sharp.

(4) If $a \in E$ is an atom then $a \leq b$ or $a \leq b^{\prime}$ for every $b \in E$.

Proof See Gudder and Greechie [5, Corollary 3.5, Corollary 4.3, proof of Theorem 5.5 and Lemma 5.2.

\section{Weak Distributivity, Maximality Property}

We will present two properties and show that an orthomodular poset with these two properties is a Boolean algebra (see [7).

Definition 4.1 An effect algebra $E$ is weakly distributive if, for every $a, b \in E, a=0$ whenever $a \wedge b=a \wedge b^{\prime}=0$.

Obviously, every Boolean algebra is weakly distributive. Let us present an example of a weakly distributive orthomodular poset that is not a Boolean algebra.

Example 4.2 Let $X_{1}, X_{2}, X_{3}, X_{4}$ be mutually disjoint infinite sets. Let us put $X=\bigcup_{i=1}^{4} X_{i}$,

$$
\begin{aligned}
E^{\prime} & =\left\{\emptyset, X_{1} \cup X_{2}, X_{2} \cup X_{3}, X_{3} \cup X_{4}, X_{4} \cup X_{1}, X\right\}, \\
E & =\left\{(A \backslash F) \cup(F \backslash A): A \in E^{\prime} \text { and } F \subset X \text { is finite }\right\} .
\end{aligned}
$$

Then $(E, \oplus, \emptyset, X)$ with $A \oplus B=A \cup B$ for disjoint $A, B \in E$ is an orthomodular poset. The orthosupplement is the set theoretic complement in $X$, the partial ordering is the inclusion.

$E$ is not a lattice because $X_{1} \wedge X_{2}$ does not exist (the set of lower bounds - the set of finite subsets of $X_{1} \cup X_{2}$ - does not have a greatest element).

Let us prove that $E$ is weakly distributive. Let $A, B \in E$ such that $A \wedge B=A \wedge B^{\prime}=\emptyset$. Since $\{x\} \in E$ for every $x \in X$, we obtain that $A \cap B=A \cap B^{\prime}=\emptyset$ and therefore $A \cap X=A \cap\left(B \cup B^{\prime}\right)=(A \cap B) \cup\left(A \cap B^{\prime}\right)=\emptyset$. Hence $A=\emptyset$.

In the sequel we will use the following statement (see also [8], Proposition 2.2).

Proposition 4.3 Let $E$ be an atomic effect algebra such that $a \leq b$ or $a \leq b^{\prime}$ for every atom $a \in E$ and for every element $b \in E$. Then $E$ is weakly distributive. 
Proof Let us suppose that $E$ is not weakly distributive and seek a contradiction. There are elements $a, b \in E$ such that $a \wedge b=a \wedge b^{\prime}=0$ and $a \neq 0$. Since $E$ is atomic, there is an atom $c \in E$ such that $c \leq a$. Since $a \wedge b=a \wedge b^{\prime}=0$, we obtain that $c \not \leq b$ and $c \not \leq b^{\prime}-\mathrm{a}$ contradiction.

Definition 4.4 An effect algebra $E$ has the maximality property if $[0, a] \cap[0, b]$ has a maximal element for every $a, b \in E$.

Let us show some examples of effect algebras with the maximality property.

Proposition 4.5 An effect algebra $E$ has the maximality property if at least one of the following conditions hold:

(1) $E$ is a lattice.

(2) $E$ is chain finite.

Proof (1) For every $a, b \in E$, the element $a \wedge b$ is a maximal (even the greatest) element of $[0, a] \cap[0, b]$.

(2) Let $a, b \in E$. According to Zorn's lemma, there is a maximal chain in $[0, a] \cap[0, b]$. According to the assumption, this chain has a maximal element and this element is also a maximal element of $[0, a] \cap[0, b]$.

Theorem 4.6 Every weakly distributive orthomodular poset with the maximality property is a Boolean algebra.

Proof See Tkadlec [7, Theorem 4.2 .

It should be noted that the above theorem cannot be generalized to orthoalgebras (see the so-called Fano plane in [1, Sect. 7).

\section{Main Results}

Let us recall two known results about the center of an effect algebra.

Theorem 5.1 (1) The center of an effect algebra is a sub-effect algebra and forms a Boolean algebra.

(2) The center of a sequential effect algebra is the set of sharp elements that commute (with respect to the sequential product) with all elements.

Proof (1) See Greechie et al. 4, Theorem 5.4.

(2) See Gudder and Greechie [5, Theorem 4.4.

Proposition 5.2 An atom in a sequential effect algebra is central iff it is sharp.

Proof It follows from the part (2) of Theorem 5.1 and from Proposition 3.3.

Proposition 5.3 Every atomic sequential effect algebra is weakly distributive. 
Proof It follows from Proposition 3.6 and Proposition 4.3

Theorem 5.4 Every atomic sequential orthoalgebra with the maximality property is a Boolean algebra.

Proof Let $E$ be an atomic sequential orthoalgebra with the maximality property. According to Proposition 3.6. $E$ is an orthomodular poset. According to Proposition 5.3, $E$ is weakly distributive. Since $E$ has the maximality property, we obtain, according to Theorem 4.6 , that $E$ is a Boolean algebra.

Corollary 5.5 Every chain finite sequential effect algebra is a Boolean algebra.

Proof Let $E$ be a chain finite sequential effect algebra. Then $E$ is atomic. According to Proposition 3.6, every atom is sharp. According to Corollary 2.6, $E$ is an orthoalgebra. According to Proposition 4.5. $E$ has the maximality property. The rest follows from Theorem 5.4

Let us remark that the last corollary was stated in Gudder and Greechie [5], Theorem 5.5(ii), with a different proof.

Theorem 5.6 Every sequential effect algebra determined by atoms such that every atom is sharp is a Boolean algebra.

Proof Let $E$ be a sequential effect algebra determined by atoms such that every atom is sharp. Let $b, c \in E$. According to Proposition 3.5, the sets of atoms dominated by $b \circ c$ and $c \circ b$ coincide. Since $E$ is determined by atoms, $b \circ c=c \circ b$. According to Theorem 5.1, the center is the set of sharp elements. According to Corollary 2.6, $E$ is an orthoalgebra, hence every element of $E$ is central. The rest follows from Theorem 5.1

The last theorem generalizes the result of Gudder and Greechie 5 that was stated for atomistic sequential orthoalgebras (see Lemma 2.2 and Example 2.3). According to this theorem, the orthomodular poset from Example 2.3 cannot be organized into a sequential effect algebra (every element in an orthomodular poset is sharp).

Theorem 5.7 The set of sharp elements of a weakly distributive lattice sequential effect algebra is a Boolean algebra.

Proof Let $E$ be a weakly distributive lattice sequential effect algebra. According to Proposition 3.6, the set $S(E)$ of sharp elements of $E$ is an orthomodular poset and a sublattice of $E$. Hence, according to Proposition 4.5, $S(E)$ has the maximality property. Moreover, since $E$ is weakly distributive, $S(E)$ is weakly distributive, too. Hence, according to Theorem 4.6. $S(E)$ is a Boolean algebra.

Corollary 5.8 The set of sharp elements of an atomic lattice sequential effect algebra is a Boolean algebra.

Proof According to Proposition 5.3 every atomic sequential effect algebra is weakly distributive. The rest follows from Theorem 5.7. 


\section{Acknowledgement}

The work was supported by the research plan of the Ministry of Education of the Czech Republic no. 6840770010.

\section{References}

1. Foulis, D. J., Bennett, M. K.: Tensor products of orthoalgebras. Order 10, 271-282 (1993)

2. Foulis, D. J., Bennett, M. K.: Effect algebras and unsharp quantum logics. Found. Phys. 24, 1331-1352 (1994)

3. Greechie, R. J.: A particular non-atomistic orthomodular poset. Commun. Math. Phys. 14, 326-328 (1969)

4. Greechie, R. J., Foulis, D., Pulmannová, S.: The center of an effect algebra. Order 12, 91-106 (1995)

5. Gudder, S., Greechie, R.: Sequential products on effect algebras. Rep. Math. Phys. 49, 87-111 (2002)

6. Pták, P., Pulmannová, S.: Orthomodular Structures as Quantum Logics. Kluwer Academic Publishers, Dordrecht (1991)

7. Tkadlec, J.: Conditions that force an orthomodular poset to be a Boolean alebra. Tatra M. Math. Publ. 10, 55-62 (1997)

8. Tkadlec, J.: Central elements of atomic effect algebras. Int. J. Theor. Phys. 44, 2257-2263 (2004) 